\title{
Movement behaviour of fish, harvesting-induced habitat degradation and the optimal size of marine reserves
}

\author{
Yousef Alqawasmeh* and Frithjof Lutscher ${ }^{\dagger}$
}

January 14, 2019

\begin{abstract}
Many models have assessed how marine reserves protect fish populations and - under certain conditions - simultaneously increase yield. Only recently have models considered the effects of fishing-induced habitat damage by assuming reduced population growth in fishing areas. Even though it is understood that fish movement patterns affect the functioning and design of marine reserves, fishing-induced changes in movement patterns, as a response to decreased habitat quality, have not been studied in this context. Our work explores how harvesting-induced movement behaviour of fish can affect optimal yield and size of a marine reserve. Our model is based on reaction-diffusion equations and recent advances in their application to strongly heterogeneous environments with sharp transitions in environmental conditions. We model movement behaviour in response to harvesting and habitat destruction via increased diffusion rates and increased preference for protected areas, and implement reduced reproduction as an effect of habitat degradation. We find an alternative mechanistic explanation for the empirical observation that high fish mobility may not decrease fish density inside

\footnotetext{
*Department of Mathematics and Statistics, University of Ottawa, Ottawa, ON, K1N6N5, Canada

${ }^{\dagger}$ corresponding author Department of Mathematics and Statistics, and Department of Biology, University of Ottawa, Ottawa, ON, K1N6N5, Canada. email: flutsche@uottawa.ca
} 
a reserve. We also find that movement-behavioural responses of fish to harvesting can decrease the economic value of protected areas and increase their conservation value. For maximum sustainable yield, we find that a low harvesting rate and small protected area are optimal when fish show a strong preference for protected areas as a response to fishing efforts. On the other hand, a high harvesting rate and a large protected area are optimal if fish respond to harvesting by a strong increase in movement rates in fishing areas.

\section{Introduction}

Fish stocks are declining worldwide, thereby threatening the survival of many fish species and decreasing fisheries' yield (Hutchings, 2000). Marine reserves or, more generally, marine protected areas (MPAs), have been suggested and established to protect stocks and secure yield through spill-over effects (Harrison et al., 2012). Both of these functions of MPAs depend on the movement behaviour of fish, but in antagonistic ways. Simply speaking, if fish tend to stay within a MPA, the chance of population persistence inside the MPA is enhanced but spill-over, and thereby potential yield outside the MPA, is reduced, and vice versa. One aspect that has previously received only marginal attention is that the establishment of an MPA and the resulting spatial pattern of fishing effort itself may influence the movement behavior of fish, thereby affecting the performance of the MPA. In this work, we present and analyze a spatially explicit model for fish population dynamics in the presence of MPAs and harvesting. In particular, we consider the feedbackdue to movement behavior - between the location and intensity of harvesting and the performance of the MPA.

Even though various types of movement patterns of fish have been described and classified (Grüss et al., 2011; White, 2015), their effect on the efficiency of MPAs is not fully understood. Based on their review of larval and fish dispersal distances, Green et al. (2015) give various rule-of-thumb guidelines for the design of networks of reserves, but detailed movement behaviour on small scales is not considered. Moreover, fishing can damage habitat by removing and altering resources and structure (Coleman and Williams, 2002; Fogarty, 2005; Hiddink et al., 2007; Olsgard et al., 2008). Such damage may not only reduce productivity and recruitment but also induce changes in the movement behaviour of fish since some species of fish adapt their movement to local conditions (Grüss et al., 2011). Since empirical work is difficult at the 
relevant scales, models play an important role in exploring these relationships and informing the design of MPAs (Botsford et al., 2001).

Neubert (2003) was the first to apply reaction-diffusion equations to questions of reserve design. He showed that MPAs arise as solutions of an optimal control problem. Under certain conditions, the MPA is located at the centre of a good habitat, which is surrounded by a hostile environment, and the transition between MPA surrounding fishing area is fairly sharp. The conditions are that the dispersal scale of the fish is not too small compared to the overall size of the habitat. Empirical data show that fishing effort often concentrates at the edge of a MPA so that the transition is indeed very sharp (see, e.g., Figure 1 in Moberg et al. (2015)). Moeller and Neubert (2013) presented the first study on how harvesting-induced reduction in productivity affects the value of MPAs. In particular, they gave conditions for a MPA to be economically advantageous - under different tax scenarios - over not having any protected areas. They considered a single reaction-diffusion equation for the density of the fish stock. They modelled the effects of habitat damage through harvesting by a reduction in net per-capita stock growth according to harvesting effort. Movement of fish was modelled by simple diffusion, independent of effort. Kelly et al. (2016) also considered a reaction-diffusion model for fish density and allowed the movement behaviour of fish to vary in space and time. This modelling framework could include harvesting-dependent movement, but the applications by Kelly et al. (2016) focus on constant diffusion and spatio-temporally varying advection to represent different water current patterns. The question of fisheries-induced movement behaviour was not addressed. So far, only one study on the effect of altered movement behaviour exist to our knowledge. Moberg et al. (2015) considered a discrete-space twopatch model and determined the evolutionarily optimal rate of movement of fish between the two patches, as well as the corresponding optimal harvesting strategy.

Langebrake et al. (2012) studied a different aspect of fish movement and MPAs. Their study was inspired by the following empirical observation. Claudet et al. (2010) measured the relative density of fish within and outside a MPA for species with different mobility. This relative density was no lower for fish with high mobility than for fish with low mobility. Theoretical models, however, predict that since individuals of species with high mobility leave the MPA more frequently, the relative density of these species within versus outside the MPA should be lower than for species with low mobility; see references in Langebrake et al. (2012). Claudet et al. (2010) speculated 
that a strong tendency to stay in the reserve could explain this effect. Langebrake et al. (2012) showed that a sufficiently strong preference for reserves in their model was indeed a mechanism by which the density inside the reserve would not decrease with mobility. Langebrake et al. (2012) did not study the implications of differential movement or habitat preference on the efficacy of marine reserves. They also considered relatively simple population dynamics of constant recruitment and linear mortality. These assumptions allowed them to analytically prove most of their results instead of relying on numerical methods.

In our work, we combine ideas from Langebrake et al. (2012) with those from Moeller and Neubert (2013) to analyze the effect of fisheries-induced habitat degradation and its movement-behaviour consequences in fish on the optimal size of a MPA and the corresponding effort. We build on our own analysis of structured population models in heterogeneous landscapes (Alqawasmeh, 2017; Alqawasmeh and Lutscher, 2019). We considerably extend the model by Langebrake et al. (2012) in three ways.

1. Instead of density-independent recruitment, we consider recruitment to be a concave function of stock size. Langebrake et al. (2012) already suggested that their model should be evaluated with non-constant recruitment. With constant recruitment, the fish stock can never become extinct; the implicit assumption is that recruits arrive from elsewhere. This setting may yield only limited insights into harvesting outcomes.

2. Instead of an unstructured population, we consider a two-stage population of immature juveniles and reproductive adults. This distinction seems necessary since some fish have relatively long immature stages, and since larger (i.e., older) fish are often selectively (or exclusively) harvested (Millar, 1992; Tsikliras and Polymeros, 2014).

3. We allow for a more general description of movement behavior at the edge of a MPA. Between a MPA and its surroundings, sharp transitions in environmental conditions, called interfaces, may occur (see above). Such sharp transitions are difficult to accommodate in classical reaction-diffusion equations. We use recent modelling advances to include interfaces and the movement behavior of individuals near them (Ovaskainen and Cornell, 2003). These conditions reflect differences in movement rates on either side of an interface and habitat preference 
by individuals. When the movement rates on either side of an interface are identical, our conditions reduce to those used by Langebrake et al. (2012), but when movement rates differ, this difference needs to be reflected in the interface conditions (Maciel and Lutscher, 2013).

Basic foraging theory would suggest that fish movement should change from exploitative to exploratory movement behaviour as resources decrease and risk increases (e.g., due to decreased habitat complexity) and that individuals should preferentially choose more suitable over less suitable habitat. In a reaction-diffusion model, we would therefore consider the diffusion coefficient to be an increasing function of habitat degradation, and thereby also of harvesting effort. Similarly, we consider habitat preference for the MPA to be increasing with harvesting effortin the surrounding area. These population and movement dynamics aspects in our model are considerably more complex than in the model by Moeller and Neubert (2013), however, our optimization approach is much simpler than their tax-based optimization in that we simply optimize yield at steady state.

We explain our model in detail in the next section. Then we study how individual behaviour of fish at interfaces affect the steady state of the model and various characteristic quantities (e.g., yield and abundance). Finally, in Section 4, we show how the optimal size of the MPA depends on the various model aspects.

\section{Model}

For the population dynamics component, we divide the population into reproductive adults and immature juveniles. Adults produce juveniles at a rate that decreases with total population density. Juveniles mature and become adults. Individuals at either stage die at a stage-specific rate. We denote the density of juveniles and adults by $u$ and $v$, respectively. These densities satisfy the equations

$$
\dot{u}=f(u, v), \quad \dot{v}=g(u, v),
$$

with

$$
f(u, v)=\frac{r v}{1+\rho_{u} u+\rho_{v} v}-\left(m+\mu_{u}\right) u, \quad g(u, v)=m u-\mu_{v} v .
$$


Here, we denote by $r$ the maximum growth rate, by $m$ the maturation rate and by $\mu_{u, v}$ the stage-specific death rate (all these rates are per-capita rates). By a simple rescaling, we may choose the weights, $\rho_{u, v}$, in the densitydependent growth rate to equal unity. Our model is a continuous-time version of the discrete-time model in Neubert and Caswell (2000). Other density-dependent effects are certainly possible, but we will focus our work on movement behaviour instead.

For the spatial aspects, we consider a habitat consisting of marine protected (MPA) and unprotected (UPA) areas. We follow previous authors and choose the simplest possible but meaningful set-up, where space is represented by the one-dimensional variable $x$, and a MPA (UPA) is an interval of length $L_{1}\left(L_{2}\right)$. Specifically, we choose the MPA to be located at $x \in\left[-L_{1} / 2, L_{1} / 2\right]$ and the UPA at $x \in\left[-L / 2,-L_{1} / 2\right] \cup\left[L_{1} / 2, L / 2\right]$ with $L=L_{1}+L_{2}$. This set-up can represent a single period of length $L$ of a periodic coastline with alternating MPAs and UPAs of the respective lengths (Botsford et al., 2001; Langebrake et al., 2012). It can also represent a single domain of length $L$ with a MPA at the center. In spatially-explicit optimal harvesting problems, the location of a MPA often happens to be at the center of a bounded domain (Moeller and Neubert, 2013; Kelly et al., 2016). We will use both points of view in our analysis.

The population-dynamics parameters in (1) are assumed constant within each habitat type but may vary between the two types. We will denote the values of the parameters by indices 1 and 2 , i.e., $\mu_{v 1}$ for the death-rate of adults in a MPA and $\mu_{v 2}$ in the UPA, and similarly for the other parameters. Harvesting in UPAs is included into the death rates. Throughout, only adults will be harvested, i.e., $\mu_{v 2}$ is replaced by $\mu_{v 2}+h$, where $h$ is the per-capita harvesting rate. Within each habitat type, individuals move randomly with stage- and habitat-specific diffusion coefficients. We denote by $u_{i}(t, x)$ and $v_{i}(t, x)$ the spatial densities of juveniles and adults in the MPA $(i=1)$ and UPA $(i=2)$. Then we have the equations

$$
u_{i t}=D_{u i} u_{i x x}+f_{i}\left(u_{i}, v_{i}\right), \quad v_{i t}=D_{v i} v_{i x x}+g_{i}\left(u_{i}, v_{i}\right)
$$

with $x \in\left[-L_{1} / 2, L_{1} / 2\right]$ for $i=1$ and $x \in\left[-L / 2,-L_{1} / 2\right] \cup\left[L_{1} / 2, L / 2\right]$ for $i=2$. Subscripts $t, x$ denote partial derivatives, subscripts $i$ denote the habitat type. Diffusion coefficients $D_{u i}, D_{v i}$ may vary between life stage and habitat type.

At an interface between MPA and UPA, a juvenile (adult) chooses to move to the MPA with some probability $\alpha_{u}\left(\alpha_{v}\right)$. Then the matching conditions 
for juveniles at $L_{1} / 2 \mathrm{read}$

$$
\begin{gathered}
u_{1}\left(t, L_{1}^{-} / 2\right)=k_{u} u_{2}\left(t, L_{1}^{+} / 2\right), \\
D_{u_{1}} \frac{\partial}{\partial x} u_{1}\left(t, L_{1}^{-} / 2\right)=D_{u_{2}} \frac{\partial}{\partial x} u_{2}\left(t, L_{1}^{+} / 2\right),
\end{gathered}
$$

with $k_{u}=\frac{\alpha_{u}}{1-\alpha_{u}} \frac{D_{u_{2}}}{D_{u_{1}}}$. Superscripts ${ }^{ \pm}$indicate one-sided limits from the right and left, respectively. The conditions at $-L_{1} / 2$ are analogous with left- and right-hand limits interchanged. The conditions for adults are the same with indices $u$ replaced by $v$. These conditions were derived by Ovaskainen and Cornell (2003); Maciel and Lutscher (2013), and their effects have since been studied intensively (Maciel and Lutscher, 2015; Alqawasmeh and Lutscher, 2019; Yurk and Cobbold, 2018).

Our focus in this work is on qualitative aspects of the positive steady state of the reaction-diffusion system in (3) with interface conditions in (4)-(5). We shall denote the steady-state densities by $u_{i}^{*}$ and $v_{i}^{*}$. Since there is generally no explicit analytical expression for this steady state (but see Section 3.2), most of our work will rely on numerical simulations. We summarize several analytical results that underlie and justify our approach here. Existence and uniqueness of solutions of a similar system was recently shown by Maciel et al. (submitted). It was also shown that the linearized system (at zero) admits a dominant eigenvalue that determines the stability of the trivial solution. The system is monotone (or order-preserving) and has bounded solutions, so that when the trivial state is unstable, there exists a (smallest) positive steady state, and this positive state is stable. Stability conditions for the trivial solution were recently given by Alqawasmeh and Lutscher (2019), together with a detailed analysis of the minimal speed of traveling waves in a periodic habitat. Since the system is invariant under the transformation $x \mapsto-x$, steady-state solutions are also symmetric, and we can reduce the system to the spatial domain $x \in[0, L / 2]$ with no-flux boundary conditions at $0, L / 2$ and interface conditions only at $L_{1} / 2$. For our numerical scheme, we simulated the time-dependent problem with a simple, fully discrete, explicit solver until the solution reached the unique (smallest) stable positive steady state.

In Figure 1, we illustrate some typical spatial profiles of the steady state and demonstrate how these profiles depend on the harvesting rate. We observe the discontinuity at the interface between MPA and UPA. The density of adults (solid lines) is decreasing in space in each patch in all cases. The 


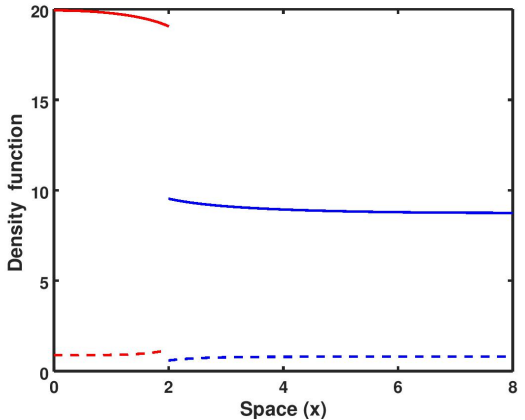

(a) $h=0.3$

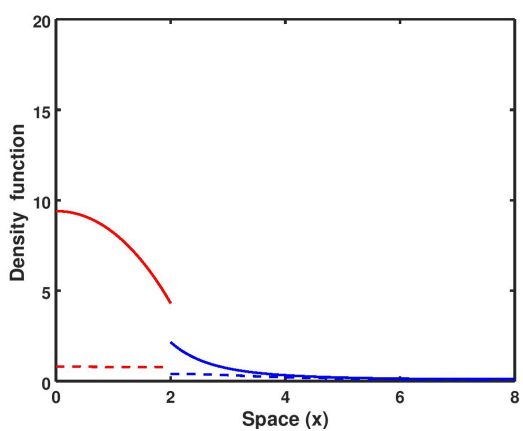

(c) $h=5$

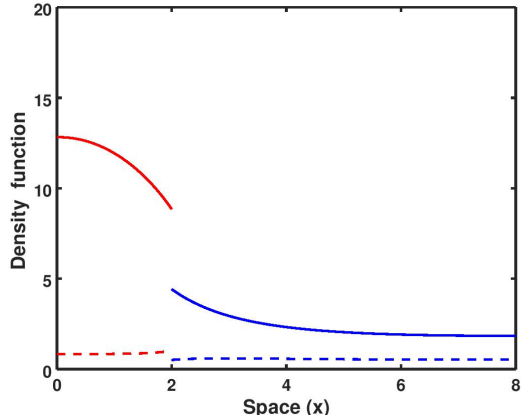

(b) $h=1.5$



(d) $h=10$

Figure 1: Steady-state solutions for juveniles (dashed) and adults (solid) in MPAs $(0<x<2)$ and UPAs $(2<x<8)$ for different values of harvesting rates $(h)$. Parameter values are: $L_{1} / 2=2, L_{2} / 2=6, r_{i}=6, m_{i}=6$, $\mu_{u_{i}}=\mu_{v_{i}}=0.25(i=1,2), D_{u_{1}}=D_{v_{1}}=1, D_{u_{2}}=D_{v_{2}}=2$, and $\alpha_{v, u}=0.5$.

density of juveniles (dashed lines) is patch-wise increasing when harvesting is small, but decreasing when harvesting is large. Overall density clearly decreases as harvesting increases. We chose no-flux boundary conditions at both ends of the interval, so that the solution corresponds to one half period of the solution on the infinite line with periodically arranged MPAs and UPAs.

Our model has a large number of parameters so that a complete analysis of all aspects is impossible. We concentrate on the movement behaviour of adults since we assume that their ability to detect environmental conditions and adjust movement behaviour is more highly developed than in juveniles. 
We will fix juvenile movement behaviour to be equal in MPA and UPA or faster in UPA (see introduction) and not consider any movement bias towards MPA in juveniles. We conducted a few numerical simulations that seemed to indicate that if juveniles' movement behaviour is similar to that of adults, the qualitative aspects of our results do not change.

\section{Individual behaviour and density patterns}

In this section, we study how the movement behaviour of individuals affects certain aspects of the population density at steady state. Specifically, we adapt the four indicators from Langebrake et al. (2012) to our model. We show, as did they, that neglecting individual movement behaviour at interfaces (i.e., setting $k_{u}=k_{v}=1$ in our model) produces patterns that are inconsistent with the empirical data from Claudet et al. (2010); see introduction. Including this movement behaviour, however, gives consistent patterns and provides mechanistic explanations for how these patterns arise.

The four indicators from Langebrake et al. (2012) are based on an unstructured population; we adjust these indicators to reflect our assumption that only adult fish are being harvested. The four quantities are as follows.

1. Adults yield is the number of mature fish caught in the UPA per unit of time, at steady state. It is given by

$$
Y=\int_{\frac{L_{1}}{2}}^{\frac{L}{2}} h v_{2}^{*}(x) d x,
$$

where $h$ represents the harvesting rate and $v_{2}^{*}(x)$ is the steady-state solution for adults in model (3) when $x \in\left[L_{1} / 2, L / 2\right]$.

2. Adults abundance in UPA is

$$
A_{0}=\int_{\frac{L_{1}}{2}}^{\frac{L}{2}} v_{2}^{*}(x) d x .
$$

3. Total adults abundance in MPA and UPA is

$$
A=A_{0}+A_{1}=A_{0}+\int_{0}^{\frac{L_{1}}{2}} v_{1}^{*}(x) d x,
$$

where $v_{1}^{*}$ is the component of the steady-state density in the MPA, $x \in\left[0, L_{1} / 2\right]$. 
4. Adults log ratio is defined as the logarithm of the ratio of the average steady-state densities

$$
R=\ln \left(\frac{A_{1} / L_{1}}{A_{0} / L_{2}}\right)
$$

We begin our investigation of these four indicators with the effect of harvesting rate, and then turn to the effects of the movement rates.

\subsection{The effects of harvesting and diffusion}

As could be expected, adults yield shows a unique maximum at some intermediate harvesting rate (Figure 2(a)). A low harvesting rate gives a low yield, whereas a high harvesting rate leads to a low steady-state density (see Figure 1), which in turn gives a low yield. As expected, abundance in fishing areas and total abundance are both decreasing with harvesting (Figure 2(b) and $(\mathrm{c}))$.

When adults have a higher movement rate than juveniles, adults abundance in UPA is higher (Figure 2(b)) but total adults abundance is lower (Figure 2(c)). The higher movement rate pushes adults from the MPA into the UPA where they are harvested so that yield increases (Figure 2(a)). As adult abundance in MPA and UPA decreases with harvesting, the log ratio increases (Figure 2(d)). Hence, the relative decrease in abundance inside the MPA is smaller than outside.

We now fix the harvesting rate and plot the four indicators as a function of diffusion rates; see Figure 3. Since there are four diffusion coefficients, we have to make choices. We concentrate on the movement of adults and keep juvenile movement rates fixed. For adults, we choose to vary $D_{v_{1}}=D$ and fix the ratio $\beta=\frac{D_{v_{1}}}{D_{v_{2}}}$. When $\beta>1$, adults move faster inside the MPA, when $\beta<1$, they move faster outside. Adults yield and abundance in fishing grounds increase with adults movement, see panels (a) and (b), whereas total abundance and log ratio decrease, see panels (c) and (d). If $\beta>1$, then adults move faster in MPAs than outside, which in turn means that adults leave the MPA more quickly and get harvested. Hence, yield and abundance in the UPA increase where as total abundance decreases.

Langebrake et al. (2012) considered the same scenario (for an unstructured population) with continuous interface conditions, i.e., $k=1$, see their Figure 3. While the shapes of their curves are qualitatively similar to ours,

the order of the three curves with respect to the ratio of diffusion rates is 


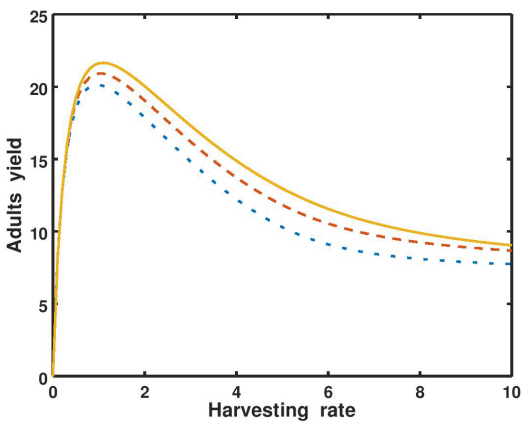

(a)

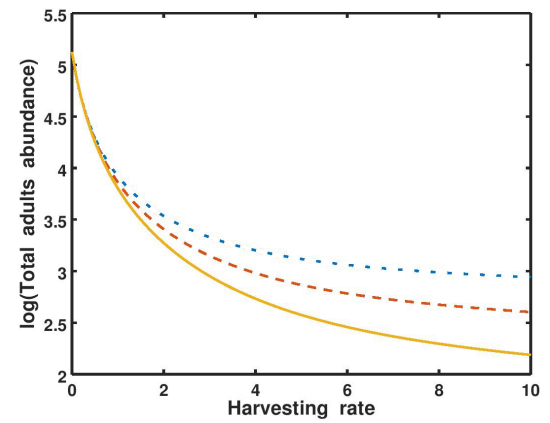

(c)

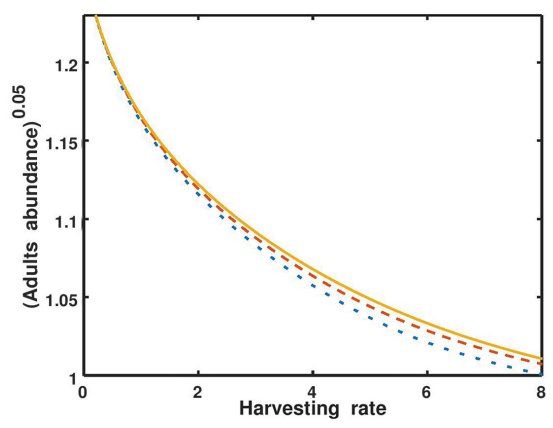

(b)

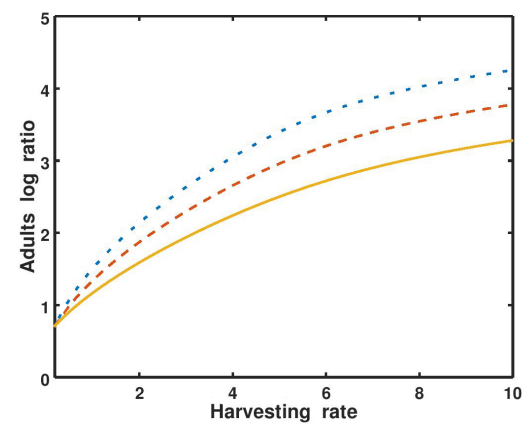

(d)

Figure 2: Effect of harvesting on adults yield $(Y)$, adults abundance $\left(A_{0}\right)$, total adults abundance $(A)$ and adults $\log$ ratio $(R)$. The three curves correspond to three different movement scenarios: juveniles move faster $\left(D_{v_{i}}=0.5 D_{u_{i}}\right.$, dotted $)$; both groups move equally $\left(D_{v_{i}}=D_{u_{i}}\right.$, dashed $)$; and adults move faster $\left(D_{v_{i}}=2 D_{u_{i}}\right.$, solid). Other parameter values are as in Figure 1, except here $m_{i}=5(i=1,2)$. 
reversed. For example, higher movement of adults inside the MPA decreased yield in their model, whereas it increases yield in our model. This difference can be explained in terms of patch preference at the interface. If adults move faster inside the MPA, i.e., $\beta=\frac{D_{v_{1}}}{D_{v_{2}}}>1$, but the density is held continuous at the interface, i.e., $k_{v}=\frac{\alpha_{v}}{1-\alpha_{v}} \frac{D_{v_{2}}}{D_{v_{1}}}=1$, then necessarily $\alpha_{v}>0.5$, i.e., adults must have a preference for the MPA. Therefore, fewer adults leave the MPA and can be harvested. We study the effect of patch preference, or movement bias at an interface in more detail.

\subsection{Biased Movement}

So far, we considered unbiased movement at the interface for both juveniles and adults (i.e., $\alpha_{u, v}=0.5$ ). In Figure $3(\mathrm{~d})$ we found that the relative densities of adults in MPAs versus UPAs decreases as adult mobility increased. The corresponding result by Langebrake et al. (2012) was the motivation for their study because it contradicts empirical findings, e.g., Claudet et al. (2010); see introduction. Claudet et al. (2010) suggested that a bias in movement toward MPAs is the main reason why higher movement rates do not lower population densities inside MPAs relative to outside. Langebrake et al. (2012) tested this idea and showed that a sufficiently high preference for MPAs resulted in an increasing relationship between the log ratio and diffusion. Their interface conditions, however, included only the term $\alpha /(1-\alpha)$ and not the quotient of the diffusion coefficients; see $k_{u}$ in (4).

The plots in Figure 4 show that the qualitative behaviour of our model is the same as that in Langebrake et al. (2012) with respect to movement bias at the interface. When adults have a small preference for MPAs $\left(\alpha_{v}=0.75\right)$, yield and abundance increase with diffusion rate (panels (a) and (b)) where as total abundance and log ratio decrease (panels (c) and (d)). When preference for MPAs is high $\left(\alpha_{v}=0.9\right)$ these trends are reversed. At some intermediate threshold value of $\alpha_{v}$, all four indicator quantities are constant with respect to $D$. When the preference for the MPA is too small, the net flux at the interface will be away from the MPA; when the preference is high, the net flux will be into the MPA. With higher diffusion rates, individuals will encounter the interface more frequently, so that its effect is stronger. As a result, abundance of adults outside the MPA (and hence also yield) will decrease with diffusion at high enough preference for the MPA.

We can calculate the threshold value explicitly by looking for steady-state 


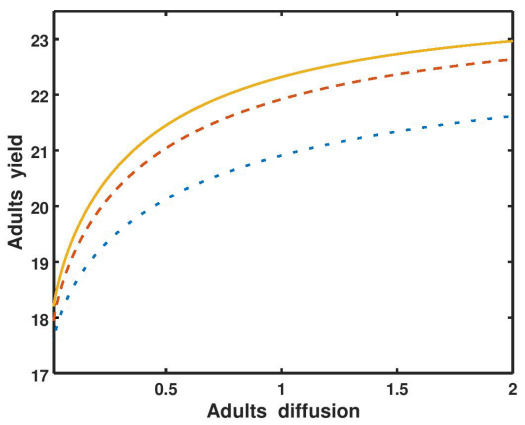

(a)

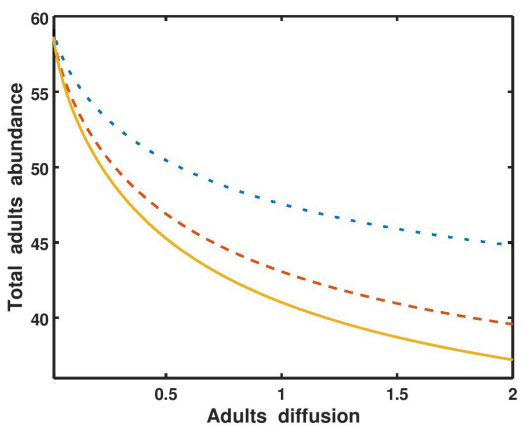

(c)

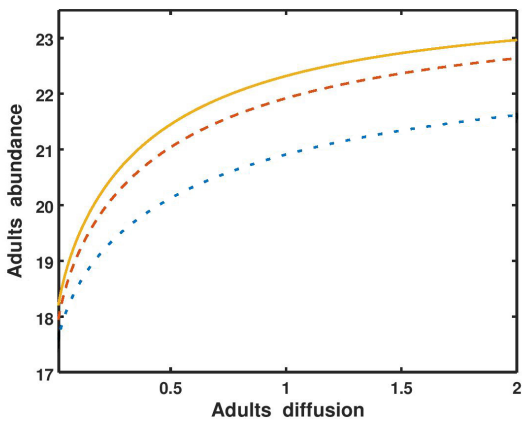

(b)

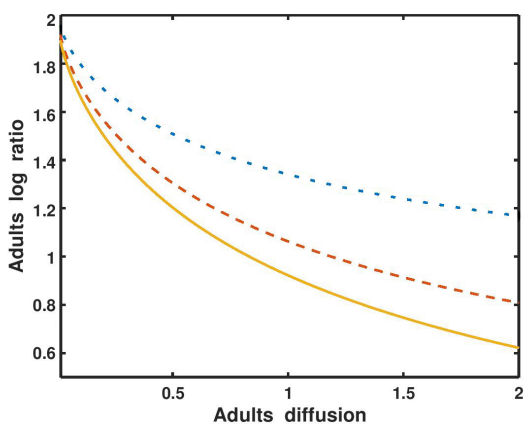

(d)

Figure 3: Effect of adults movement on the four indicator quantities. The three curves correspond to different values of the ratio $\beta=\frac{D_{v_{1}}}{D_{v_{2}}}$. When adults move faster in MPAs ( $\beta>1$, solid), $Y$ and $A_{0}$ are higher (top panels) than when movement is equal ( $\beta=1$, dashed) whereas $A$ and $R$ are lower (bottom panels). When adults move faster in UPAs $(\beta<1$, dotted), the order is reversed. We chose $h=1$ and all other parameter values as in Figure 2 . 


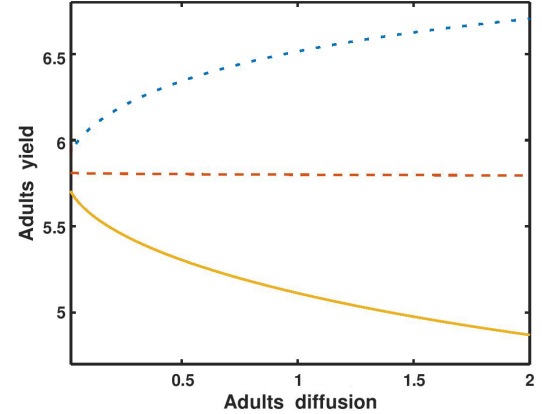

(a)

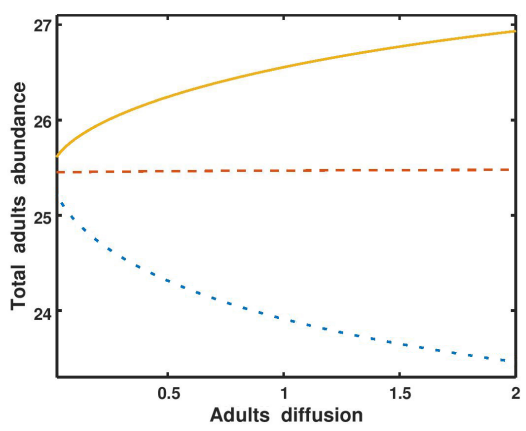

(c)

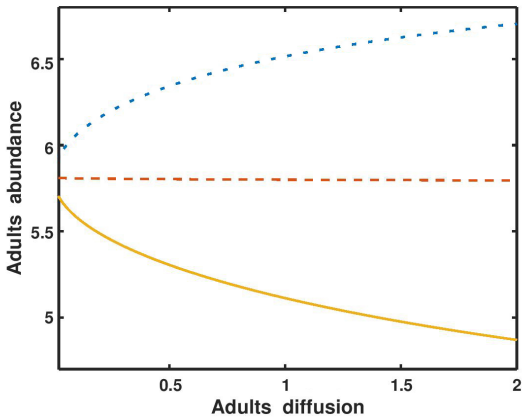

(b)

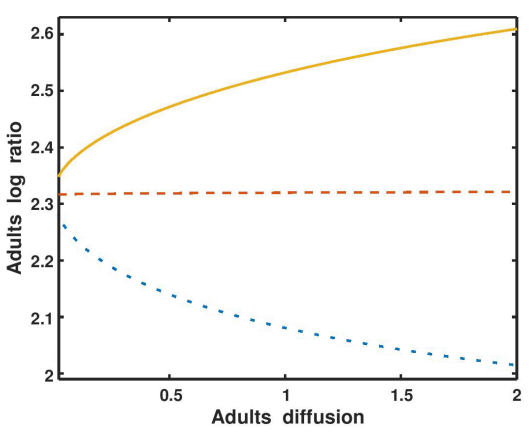

(d)

Figure 4: Effect of adults movement on the four indicator quantities. The three curves correspond to different values of adults habitat preference rate, $\alpha_{v}$. When preference for MPAs is weak $\left(\alpha_{v}=0.75\right.$, dotted), $Y$ and $A_{0}$ are increasing functions of $D$, whereas $A$ and $\log$ ratio are decreasing. When preference is strong $\left(\alpha_{v}=0.9\right.$, solid $)$ the trends are reversed. At some intermediate threshold ( $\alpha_{v}^{*}=5 / 6$, dashed), the quantities are independent of $D$. Parameter values are $r_{i}=2.9, \alpha_{u}=0.5, \beta=\frac{D_{v_{1}}}{D_{v_{2}}}=0.5$ and $h=1$. Other parameters are as in the previous figure. 
solutions for which each population is spatially constant in each patch type. Diffusion does not affect spatially constant solutions, which also automatically satisfy the no-flux boundary conditions at $x=0$ and $x=L / 2$. The matching condition at the interface $x=L_{1} / 2$ will then give us the threshold value for the parameter.

A steady-state solution that is constant in the MPA satisfies

$$
\frac{r_{1} v_{1}^{*}}{1+u_{1}^{*}+v_{1}^{*}}-\left(m_{1}+\mu_{u_{1}}\right) u_{1}^{*}=0, \quad m_{1} u_{1}^{*}-\mu_{v_{1}} v_{1}^{*}=0 .
$$

We find the explicit expressions

$$
v_{1}^{*}=\left(\frac{r_{1} m_{1}}{\mu_{v_{1}}\left(m_{1}+\mu_{u_{1}}\right)}-1\right)\left(\frac{m_{1}}{m_{1}+\mu_{v_{1}}}\right) \quad \text { and } \quad u_{1}^{*}=\frac{\mu_{v_{1}}}{m_{1}} v_{1}^{*} .
$$

We obtain analogous expressions for $u_{2}^{*}$ and $v_{2}^{*}$ in the UPA by replacing indices 1 with 2 and $\mu_{v_{1}}$ with $\mu_{v_{2}}+h$. We combine these expressions with the matching condition at the interface to get

$$
u_{1}^{*}=k_{u} u_{2}^{*}=k_{u}\left(\frac{\mu_{v_{2}}+h}{m_{2}}\right) v_{2}^{*}
$$

and

$$
u_{1}^{*}=\frac{\mu_{v_{1}}}{m_{1}} v_{1}^{*}=k_{v}\left(\frac{\mu_{v_{1}}}{m_{1}}\right) v_{2}^{*} .
$$

Equating these two conditions and substituting the expressions for $k_{u, v}$, we find that steady state solutions are piecewise constant if and only if

$$
\frac{\alpha_{u}}{1-\alpha_{u}} \frac{D_{u_{2}}}{D_{u_{1}}}\left(\frac{\mu_{v_{2}}+h}{m_{2}}\right)=\frac{\alpha_{v}}{1-\alpha_{v}} \frac{D_{v_{2}}}{D_{v_{1}}}\left(\frac{\mu_{v_{1}}}{m_{1}}\right)
$$

Substituting parameter values from Figure 4 into this formula and solving for $\alpha_{v}$, we obtain the threshold value $\alpha_{v}^{*}=\frac{5}{6}$ that corresponds to the constant dashed lines in the Figure.

Hence, our model supports the hypothesis by Claudet et al. (2010) that a sufficiently strong movement bias towards a MPA produces density patterns that are consistent with empirical observations; see introduction. These results are the same as those by Langebrake et al. (2012), which shows that they are robust with respect to several model assumptions. Even in our more complex, stage-structured model, the threshold value of movement bias that leads to the correct qualitative behaviour can be calculated explicitly. 
Langebrake et al. (2012) used different diffusion coefficients inside and outside MPAs in their model but did not include the diffusion coefficients into the matching conditions of the density at the interface. Numerous studies report habitat specific movement behaviour of fish (Grüss et al., 2011), and much empirical work on patch preferences is available in terrestrial systems (Schultz and Crone, 2001; Reeve and Cronin, 2010). Maciel and Lutscher (2013) demonstrated that several population-dynamic aspects of these reaction-diffusion equations in patchy landscapes are consistent with intuition only when the diffusion coefficients are included into the interface conditions. With the inclusion of diffusion rates in our matching conditions (parameters $k_{u, v}$ ), we can also obtain a critical diffusion ratio, $\beta^{*}$, from solving formula (14) for $\beta=D_{v_{1}} / D_{v_{2}}$.

Figure 5 shows that for $\beta>\beta^{*}, Y$ and $A_{0}$ are increasing functions of diffusion of adults whereas $A$ and $\log$ ratio are decreasing, i.e., inconsistent with the observations from Claudet et al. (2010); see introduction. For $\beta<$ $\beta^{*}$, the patterns are reversed and consistent with observations. For $\beta=\beta^{*}$, the quantities are independent of diffusion of adults.

Hence, we found a second mechanism that explains the empirical observation that relative densities inside versus outside of an MPA need not decrease with fish mobility, as simple theoretical models predict. Namely, if the ratio of the movement rates is relatively low, i.e., fish move faster outside than inside an MPA, then relative fish densities increase as movement rates increase as (absolute) diffusion rates increase. Moreover, as the ratio of movement rates decreases, the threshold value of habitat preference $\left(\alpha_{v}^{*}\right.$ above) decreases. Hence, the two mechanisms (high preference for MPA and relatively lower diffusion rate in MPA) act synergistically.

\subsection{Harvesting-dependent movement behaviour}

Habitat characteristics such as degradation or noise levels arguably depend on the intensity of harvesting. Consequently, the behavioural response of fish to harvesting could depend on harvesting levels. We use our model to explore how harvesting-dependent movement affects yield and abundance. Specifically, we assume that movement rates outside MPAs and preference for MPAs increase as harvesting increases. In this section, we only consider a movement-behaviour response to harvesting; reproduction is unaffected. In the next section, we will also include a feedback of harvesting on reproduction. 


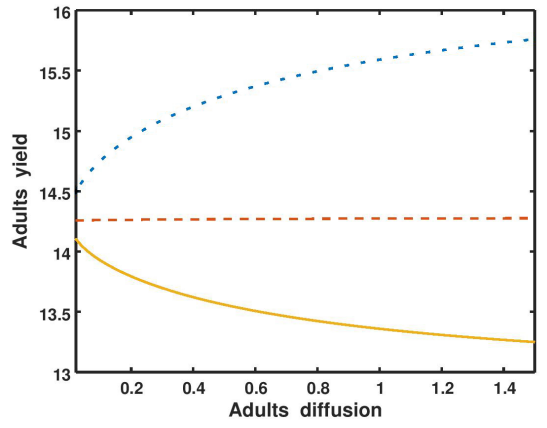

(a)

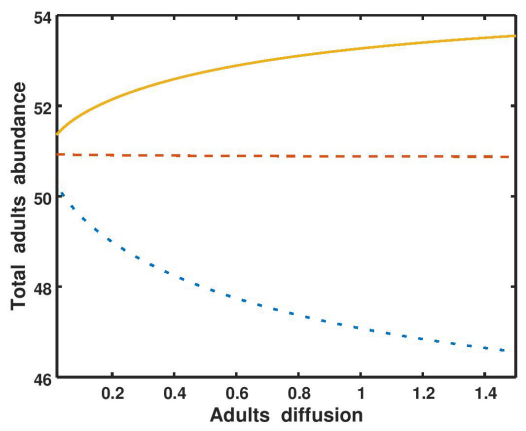

(c)

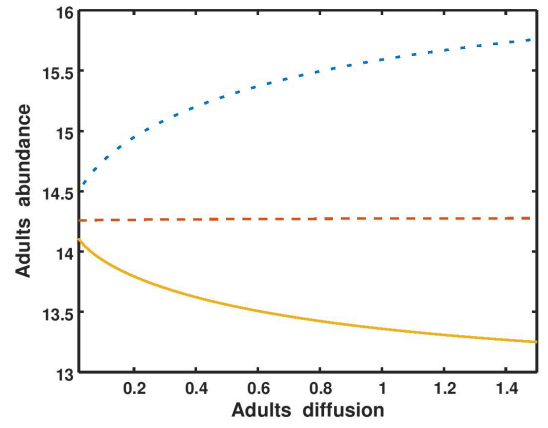

(b)

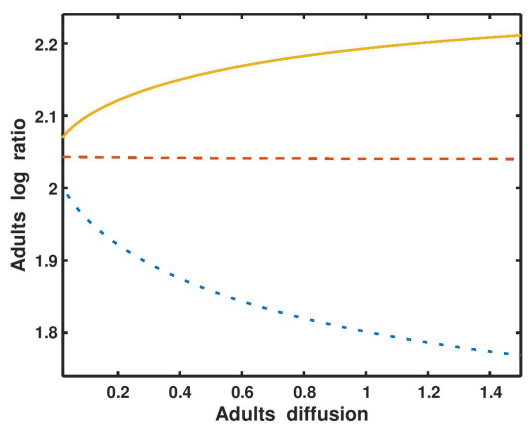

(d)

Figure 5: Effect of adults movement on the four indicator quantities. The three curves correspond to different ratios of diffusion coefficients $\beta=$ $D_{v_{1}} / D_{v_{2}}$. When the ratio is small $\left(\beta=\frac{1}{9.5}\right.$ solid), $Y$ and $A_{0}$ are decreasing functions of $D$, whereas $A$ and $\log$ ratio are increasing. When the ratio is large $\left(\beta=\frac{1}{5}\right.$ dotted) the trends are reversed. At some intermediate threshold $\left(\beta^{*}=\frac{1}{7.5}\right.$, dashed), the quantities are independent of $D$. Parameter values are: $r_{i}=5.2, m_{i}=5, D_{u_{1}}=5, D_{u_{2}}=7.5, \alpha_{u}=0.5=\alpha_{v}$ and as in the previous figure, unless otherwise noted. 
We consider the following simple relations for adults habitat preference and diffusion coefficients in fishing areas

$$
\alpha_{v}(h)=1-\frac{e^{-s h}}{2}, \quad h \geq 0
$$

and

$$
D_{v_{2}}(h)=D_{\max }-\left(D_{\max }-D_{v_{1}}\right) e^{-\tilde{s} h}, \quad h \geq 0,
$$

for some 'sensitivity' parameters $s, \tilde{s} \geq 0$ and $D_{\max }>D_{v_{1}}$. Without harvesting $(h=0)$, fish move at the same rate inside and outside MPAs and have no habitat preference. As fishing activities increase, adults mobility in fishing grounds and preference to MPAs increase.

In Figure 6, we illustrate how our four indicators are affected by harvestingdependent movement (compare Figure 2). We consider three scenarios: (i) only habitat preference depends on harvesting rate $(s>0, \tilde{s}=0)$; (ii) only movement rates depend on harvesting rate $(s=0, \tilde{s}>0)$; and (iii) both processes depend on harvesting rate $(s, \tilde{s}>0)$. We did not include the curves of the null model $(s=\tilde{s}=0)$ because they cannot be distinguished from the curves in scenario (ii).

We find that if (at least) habitat preference depends on harvesting rate, yield and abundance decrease whereas total abundance and log ratio increase compared to when there is no dependence. In other words, when fish respond to harvesting by a showing a high preference for staying in the MPA, then yield decreases and therefore the economic value of the MPA might decrease. The effect is more pronounced when diffusion rates in UPAs increase with harvesting as well. The effect can be so strong that total adult abundance may even increase with harvesting at very high harvesting rates (Figure 6, panel c). The mechanism behind this effect is similar to previous cases: With increased harvesting, adults will increasingly stay in or move to the MPA and

not be available for fishing. Under these conditions then, what is the optimal harvesting strategy?

\section{Maximum sustainable yield}

In this final section, we ask which combination of MPA size and harvesting rate results in the maximum sustainable yield at steady state. In addition to the scenario in the previous section, we now include a feedback of harvestinginduced habitat degradation on population reproduction. 


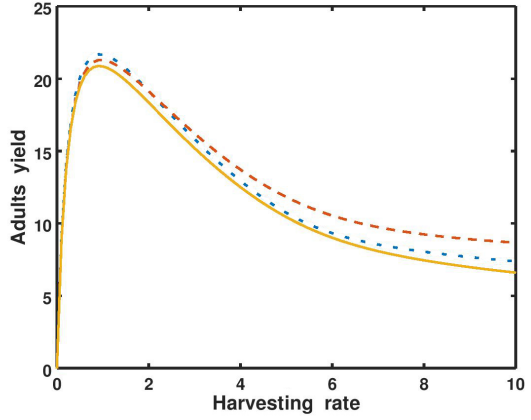

(a)

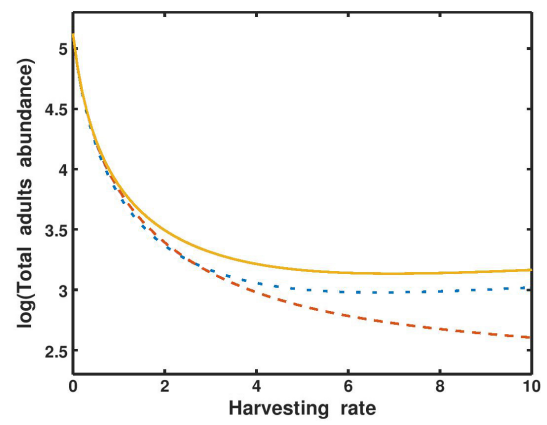

(c)

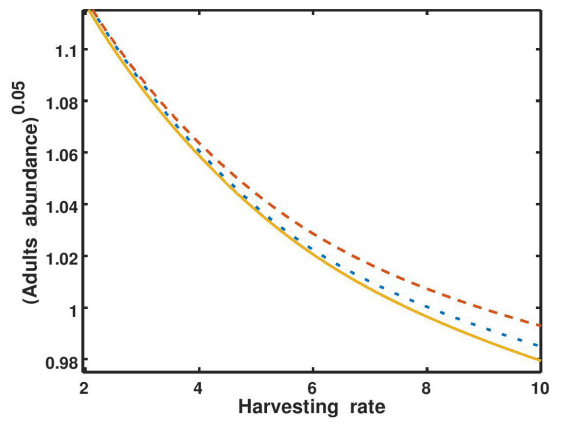

(b)

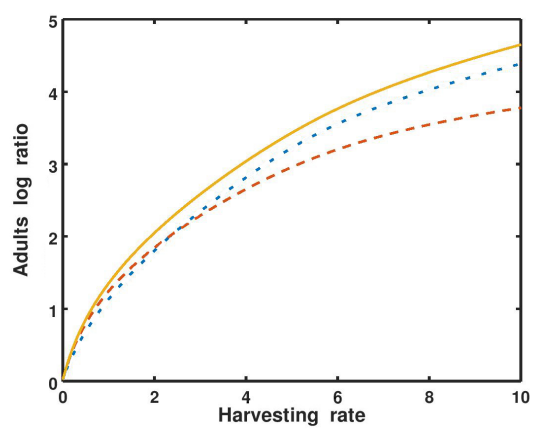

(d)

Figure 6: Effect of harvesting on adults yield $(Y)$, adults abundance $\left(A_{0}\right)$, total adults abundance $(A)$ and adults $\log$ ratio $(R)$. The three curves correspond to three different scenarios described in the text. (i) Only habitat preference depends on harvesting rate: $s=0.1, \tilde{s}=0$ (dotted). (ii) Only movement rates depend on harvesting rate $s=0, \tilde{s}=1$ (dashed). (iii) Both processes depend on harvesting rate: $s=0.1, \tilde{s}=1$ (solid). Parameter values are: $D_{u_{1}}=D_{u_{2}}=D_{v_{1}}=1, D_{\max }=2$, and as in Figure 2 unless otherwise noted. 
The population dynamics equations are as in (3). However, harvestinginduced habitat degradation now also leads to decreased productivity in the UPA. There are many ways in which this decrease can be modelled. We choose to replace the reproduction term $r_{2} v_{2}\left(1+u_{2}+v_{2}\right)^{-1}$ by

$$
r_{2} v_{2}\left(1+(1+\hat{s} h)\left(u_{2}+v_{2}\right)\right)^{-1}
$$

for some sensitivity parameter $(\hat{s}>0)$. This term could reflect an increase in intra-specific competition in the UPA that is degraded by harvesting rate. The spatial set-up in this section is as follows. We consider the interval [ $\left.-L_{1} / 2, L_{1} / 2\right]$ as a single MPA of length $L_{1}$ surrounded by a UPA of length $L_{2} / 2$ on either side. The total length, $L=L_{1}+L_{2}$ is fixed. The interface conditions at $\pm L_{1} / 2$ are exactly as in (4) and (5) with habitat preference and movement rates of adults depending on harvesting rate according to (15)(16). The difference to the previous scenario is that we now choose hostile boundary conditions at the end of the UPA at $x= \pm L / 2$. This choice follows previous work by Moeller and Neubert (2013). Mathematically, the system is equivalent to a system on the interval $[0, L / 2]$ with no-flux conditions at $x=0$. We use MPA length, $L_{1}$, and harvesting rate, $h$, as our free parameters.

When the MPA is small, its productivity for the UPA is small, and consequently, yield is low for a given harvesting rate. When the MPA is large, the UPA is small and therefore yield is as well. We use our numerical schemes to find the optimal size of the MPA and the corresponding maximum yield and harvesting rate for different values of $s, \tilde{s}$ and $\hat{s}$.

The top plots in Figure 7 show how the maximum sustainable yield (MSY, solid) arises for intermediate length of the MPA while the corresponding harvesting rate (dashed) increases with MPA length. The bottom plots show how MSY depends on the length of the MPA and the harvesting rate. Habitat preference of adults $\left(\alpha_{v}\right)$ is more sensitive to harvesting in the plots on the right, so that MSY and corresponding harvesting rate decrease.

Three parameters measure the extent to which harvesting affects the three processes that we are interested in:

1. $s$ measures how sensitive adults preference for MPA is to harvesting;

2. $\tilde{s}$ measures how sensitive adults mobility in the fishing grounds is to harvesting;

3. $\hat{s}$ measures how sensitive reproduction is to the damage in the habitat. 


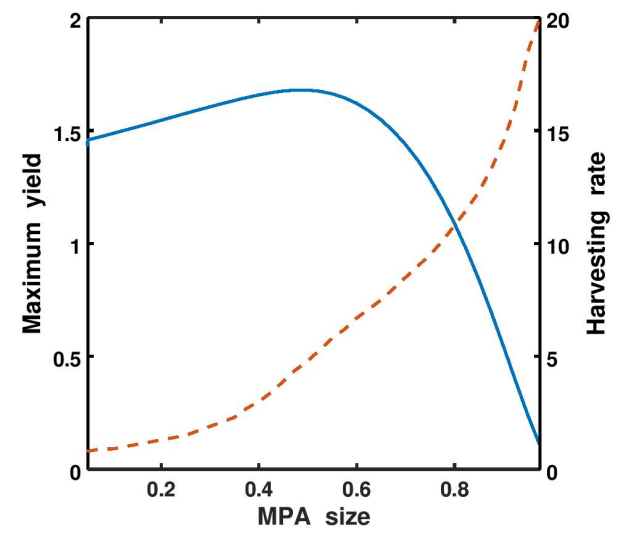

(a)

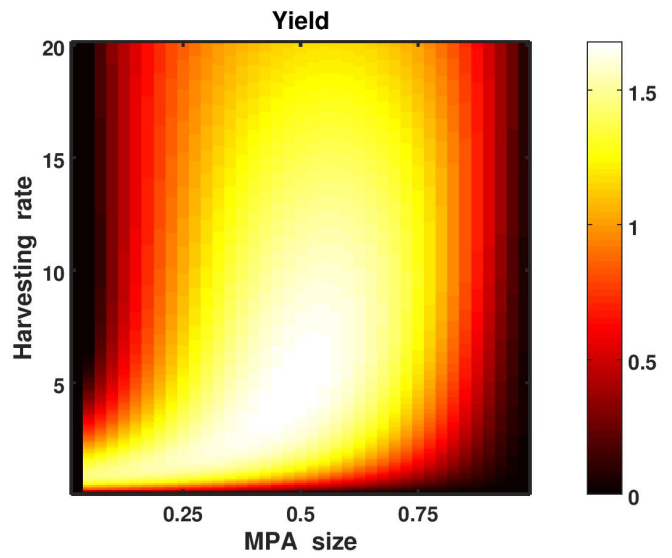

(c)

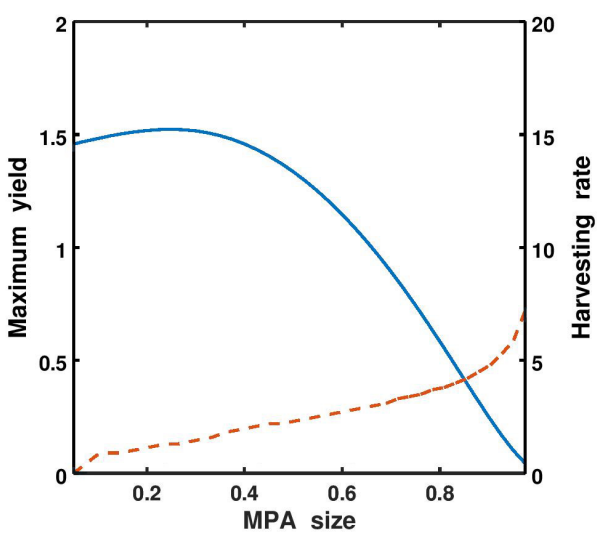

(b)

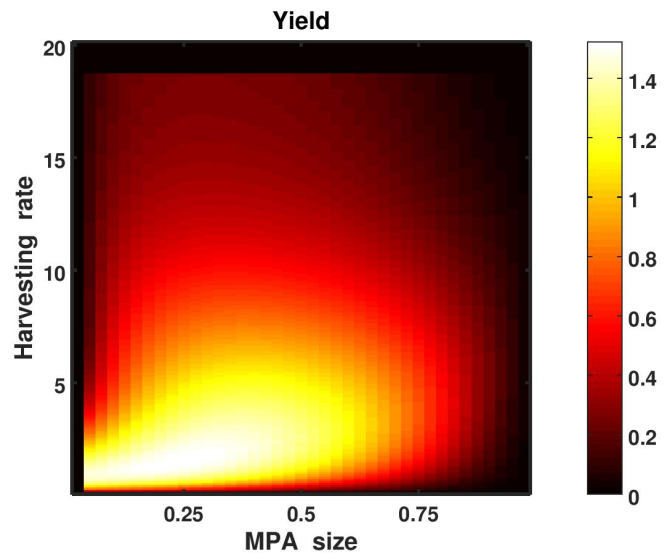

(d)

Figure 7: Maximum yield and harvesting rate as function of MPA length $\left(L_{1}\right)$. Top row: Maximum yield (solid, scale on left) and corresponding harvest rate (dashed, scale on right). Bottom row: Yield (color bar on right) as a function of harvest rate and MPA size. Left column: sensitivity of adult preference to MPA is small $(s=0.1)$. Right column: sensitivity is large $(s=0.3)$. Other parameter values are $L_{2}=1-L_{1}, m_{i}=5, r_{i}=6, \mu_{u_{i}}=\mu_{v_{i}}=0.25$, $D_{u_{1}}=D_{v_{1}}=D_{u_{2}}=0.1, D_{\max }=2, \tilde{s}=\hat{s}=0.1$ and $\alpha_{u}=0.5$. 
Table 1 shows how maximum yield, MPA size and corresponding harvesting rate vary with changes in these three sensitivities. In addition, we calculated total effort as the product of the optimal harvesting rate with the length of the UPA $\left(L_{2}=1-L_{1}\right)$.

We observe that MSY decreases with sensitivity to harvesting in each of the three parameters. However, whereas MPA size and optimal harvesting rate decrease with sensitivity of preference for MPA $(s)$ and of growth rate $(\hat{s})$, they both increase with sensitivity of movement rates $(\tilde{s})$. Total effort also decreases in the first two cases but decreases in the last. When $s$ increases, adults tend to stay in the MPA more and cannot be harvested. A smaller MPA length is sufficient to sustain the stock and a smaller harvesting rate is required to entice the fish to leave the MPA. While the lower harvesting rate is distributed over a larger region, the total effort decreases. When $\hat{s}$ increases, reproduction outside the MPA decreases more severely with harvesting. Smaller harvesting rates minimize the negative impact. A smaller MPA is sufficient. As before, total effort decreases. When $\tilde{s}$ increases, adults tend to move faster in UPA. This increased movement rate can push them back to the MPA or to the hostile boundary where they leave the system. Harvest rate has to be increased to catch fish before they leave the UPA. This requires a larger MPA to sustain the stock. Despite the fact that the length of the UPA decreases, the total effort increases.

In summary, we found - unsurprisingly - that increased sensitivity to harvesting in the three focus aspects decreased the MSY of the fishery, but the details differ. If fish react to harvesting mostly by increasing movement rates in UPA, then the best strategy is to harvest at a high rate so that fish get caught before they leave, but this then requires a large MPA to sustain the stock. If fish react to harvesting mostly by high preference for the MPA or by strongly reduced reproduction, then the best strategy is to harvest at a low rate, which, in turn, requires only a smaller MPA.

\section{Discussion}

Marine protected areas can protect fish stocks that are declining under increasing fishing pressure (Mosquera et al., 2000) and can also support fisheries in adjacent areas via spill-over effects (Hilborn et al., 2004). From the intense debate on the ecological and economic implications of MPAs (Bohnsack, 1993; Mosquera et al., 2000; Botsford et al., 2001; Roberts et al., 2001; 
Table 1: Maximum yield, MPA size, harvesting rate and total effort as functions of sensitivity parameters: (a) with $\tilde{s}=\hat{s}=0.1$, (b) with $s=0.2$ and $\hat{s}=0.1$, and (c) with $s=0.2$ and $\tilde{s}=0.1$. Other parameter values are as in Figure 7 .

(a)

\begin{tabular}{|c|c|c|c|c|}
\hline$s$ & Maximum Yield & MPA Size & Harvesting Rate & Total Effort \\
\hline \hline 0.1 & 1.6791 & 0.475 & 4.4 & 2.31 \\
\hline 0.2 & 1.5716 & 0.35 & 2.1 & 1.365 \\
\hline 0.3 & 1.5231 & 0.25 & 1.4 & 1.05 \\
\hline
\end{tabular}

(b)

\begin{tabular}{|c|c|c|c|c|}
\hline$\tilde{s}$ & Maximum Yield & MPA Size & Harvesting Rate & Total Effort \\
\hline \hline 0.1 & 1.5716 & 0.35 & 2.1 & 1.365 \\
\hline 0.3 & 1.4318 & 0.40 & 2.4 & 1.44 \\
\hline 0.5 & 1.3551 & 0.425 & 2.7 & 1.5525 \\
\hline
\end{tabular}

(c)

\begin{tabular}{|c|c|c|c|c|}
\hline$\hat{s}$ & Maximum Yield & MPA Size & Harvesting Rate & Total Effort \\
\hline \hline 0.1 & 1.5716 & 0.35 & 2.1 & 1.365 \\
\hline 0.2 & 1.5063 & 0.30 & 1.8 & 1.26 \\
\hline 0.3 & 1.4579 & 0.275 & 1.6 & 1.16 \\
\hline
\end{tabular}


Gell and Roberts, 2003; Gerber et al., 2003; Lester et al., 2009), it is clear that dispersal patterns of fish affect the function of a MPA and its optimal design (Lockwood et al., 2002; Almany et al., 2013). With a single MPA, a sufficient number of recruits must remain within the MPA to ensure the persistence of the stock, whereas a large number of exports to fishing areas increases the yield. For networks of reserves, the persistence condition is different; see Hastings and Botsford (2006). While many different dispersal patterns for fish have been documented (White, 2015; Grüss et al., 2011), theoretical studies on potential consequences for the efficacy of MPAs in this direction are rare; see e.g., Green et al. (2015). Fishing-induced habitat degradation arises in many ways (Coleman and Williams, 2002; Fogarty, 2005; Hiddink et al., 2007), most obviously in bottom trawling (Olsgard et al., 2008), but has only recently been included in theoretical studies of optimal design of MPAs (Moeller and Neubert, 2013). When fish adjust their movement behaviour to local habitat quality, then fishing itself has the potential to alter movement behaviour, and thereby the functioning and optimal design of MPAs. In this work, we presented a first theoretical model to evaluate some of these feedback effects.

Our model is a substantial extension of the model by Langebrake et al. (2012). We include age structure, density dependence, and the complete interface conditions from Ovaskainen and Cornell (2003). Our first result is the confirmation that the results by Langebrake et al. (2012) continue to hold in our model, and are therefore robust to model formulation. Our second finding is that differential movement rates between MPA and UPA provide an alternative mechanism to resolve the difference between the theoretical prediction and empirical observation from Claudet et al. (2010) about relative densities of fish inside and outside MPAs; see introduction. In a second step, we include feedbacks from harvesting on (i) movement behaviour, and (ii) also on reproduction. At first, we show that even if harvesting only affects movement behaviour (i.e., increased preference for MPA and increased diffusion rate in UPA), a MPA may lose some of its economic value but enhance its conservation value (Figure 6). Finally, we determined the optimal size of a MPA and optimal harvesting rate in terms of MSY when harvesting also negatively impacts reproduction. We showed how - depending on which feedback mechanism is the strongest - the optimal state could be higher harvesting rates and a larger MPA or lower rates and a smaller MPA.

Our modelling approach is based on reaction-diffusion equations, which have been used successfully in many areas of spatial ecology in general and 
in marine reserve design in particular (Neubert, 2003; Moeller and Neubert, 2013; Kelly et al., 2016). A common feature of the optimal control problems studied by these authors is that solutions have fairly sharp interfaces between fished and unfished areas, i.e., the fishing effort changes on a scale much smaller than the mean dispersal distance of the fish. In that case, it is reasonable to assume that fish can exhibit movement preference at such an interface, and that the movement preference would depend on the difference in habitat quality. Our model includes all these features and mechanisms, i.e., it allows fish to adjust movement behaviour in the UPA and at the interface according to habitat quality, and it can account for degradationreduced population growth.

We did not cast our question into an optimal control problem, but asked the simpler question of maximizing yield. Still, we found that the MSY occurred when a MPA was present. However, comparing the two top plots in Figure 7, it is conceivable that when the sensitivity of preference with respect to harvesting is very high, a reserve may not be optimal. When fish react to harvesting-induced damage by (almost) not leaving the MPA at all, then there is no spill-over effect and hence no positive effect on yield. It is interesting to note that the increase in mobility outside the reserve and the preference for the MPA at the interface have opposite effects on the optimal size and effort (Table 1). When fish are highly mobile outside the MPA, they may return to the MPA or leave the entire area more quickly. In the second case, they are lost from the population. Therefore, a high effort is put on catching most fish that leave the MPA before they leave the entire domain. It would be interesting to study whether and how the latter result changes when the boundary conditions are not hostile, as in the model by Kelly et al. (2016), and to apply the optimal control framework to our model.

Our model assumes that harvesting, where it is allowed, is uniform. We make this assumption since it often arises under certain conditions from optimal control models that do not make these assumptions (Neubert, 2003). It is a relatively straight forward extension to choose, say, two different harvesting levels outside an MPA, as they arise as nearly optimal under other conditions (Neubert, 2003). As long as the interface region where parameter values change is small, our model can be adapted. When gradients in parameter values are less steep so that there is no sharp interface, our approach does not apply. Instead of habitat preference at an interface, one would have to implement a preferred movement direction ("advection" or "taxis") over a larger region in order to model the effect that fish move as to stay in 
high-quality habitat. The modelling approach by Kelly et al. (2016) could be adapted to that case. The difference between the two comes down to a question of scales: does the environment change on scales that are small or large compared the mean dispersal distance of the fish? Bottom trawling creates disturbances that have relatively sharp edges; other effects such as noise and pollution are much more gradual.

It remains to be seen to what extent and how our modelling approach can be combined with an optimal control framework. In such a framework, optimal effort solutions often show the phenomenon of "fishing the line", where fishing efforts concentrate at the boundary of the reserve to catch all the fish that are leaving the reserve. Such a concentration of effort leads to a large change in model parameters on a very small spatial scale, but does not give piecewise constant parameter functions. It might be possible to formulate interface conditions for very narrow bands at the MPA boundary and study their effects. It is even possible the high concentration of fishing effort at the perimeter of an MPA would deter fish from leaving the MPA. A model that includes our movement-behaviour responses of fish to such a high concentration of effort might uncover scenarios where the "fishing the line"-strategy is not optimal.

\section{Acknowledgement}

YA and FL thank Michael Neubert for inspiring discussions. FL gratefully acknowledges funding by the Natural Sciences and Engineering Research Council of Canada through the Discovery Grants program (RGPIN-2016-04759) and a Discovery Accelerator Supplement (RGPAS-2016-492872). We also thank two reviewers for their valuable comments and suggestions.

\section{References}

Almany, G., Hamilton, R. J., Bode, M., Matawai, M., Potuku, T., SaenzAgudelo, P., Planes, S., Berumen, M., Rhodes, K., Thorrold, S., Russ, G., and Jones, G. (2013). Dispersal of grouper larvae drives local resource sharing in a coral reef fishery. Current Biology, 23, 626-630.

Alqawasmeh, Y. (2017). Models for Persistence and Spread of Structured Populations in Patchy Landscapes. Phd thesis, University of Ottawa, https://ruor.uottawa.ca/handle/10393/36845. 
Alqawasmeh, Y. and Lutscher, F. (2019). Persistence and spread of stagestructured populations in heterogeneous landscapes. Journal of Mathematical Biology. https://doi.org/10.1007/s00285-018-1317-8

Bohnsack, J. (1993). Marine reserves: they enhance fisheries, reduce conflicts, and protect resources. Oceanus, 36(1), 63.

Botsford, L., Hastings, A., and Gaines, S. (2001). Dependence of sustainability on the configuration of marine reserves and larval dispersal distance. Ecology Letters, 4(2), 144-150.

Claudet, J., Osenberg, C., Domenici, P., Badalamenti, F., Milazzo, M., Falcon, J., Bertocci, I., Benedetti-Cecchi, L., Garcia-Charton, J.-A., Goni, R., Borg, J., Forcada, A., Lucia, G. D., Perez-Ruzafa, A., Afonso, P., Brito, A., Guala, I., Direach, L., Sanchez-Jerez, P., Somerfield, P. J., and Planes, S. (2010). Marine reserves: fish life history and ecological traits matter. Ecological Applications, 20(3), 830-839.

Coleman, F. and Williams, S. (2002). Overexploiting marine ecosystem engineers: potential consequences for biodiversity. Trends in Ecology and Evolution, 17(1), 40-44.

Fogarty, M. (2005). Impacts of fishing activities on benthic habitat and carrying capacity: approaches to assessing and managing risk. In American Fisheries Society Symposium, volume 41, page 769.

Gell, F. and Roberts, C. (2003). Benefits beyond boundaries: the fishery effects of marine reserves. Trends in Ecology and Evolution, 18(9), 448455 .

Gerber, L., Botsford, L., Hastings, A., Possingham, H., Gaines, S., Palumbi, S., and Andelman, S. (2003). Population models for marine reserve design: A retrospective and prospective synthesis. Ecological Applications, 13, 4764 .

Green, A.L., Maypa, A.P., Almany, G.R., Rhodes, K.L., Weeks, R., Abesamis, R.A., Gleason, M.G., Mumby, P.J., and White, A.T. (2015). Larval dispersal and movement patterns of coral reef fishes, and implications for marine reserve network design. Biol. Rev., 90, 1215-1247. 
Grüss, A., Kaplan, D., Guénette, S., Roberts, C., and Botsford, L. (2011). Consequences of adult and juvenile movement for marine protected areas. Journal of Biological Conservation, 144, 692-702.

Harrison, H., Williamson, D., Evans, R., Almany, G., Thorrold, S., Russ, G., Feldheim, K., van Herwerden, L., Planes, S., Srinivasan, M., Berumen, M., and Jones, G. (2012). Larval export from marine reserves and the recruitment benefit for fish and fisheries. Current Biology, 22(11), 10231028.

Hastings, A. and Botsford, L.W. Persistence of spatial populations depends on returning home. PNAS, 103(15), 6067-6072.

Hiddink, J., Jennings, S., and Kaiser, M. (2007). Assessing and predicting the relative ecological impacts of disturbance on habitats with different sensitivities. Journal of Applied Ecology, 44(2), 405-413.

Hilborn, R., Stokes, K., Maguire, J., Smith, T., Botsford, L., Mangel, M., Orensanz, J., Parma, A., Rice, J., Bell, J., Cochrane, K., Garcia, S., Hall, S., Kirkwood, G., Sainsbury, K., Stefansson, G., and Walters, C. (2004). When can marine reserves improve fisheries management? Ocean and Coastal Management, 47, 197-205.

Hutchings, J. (2000). Collapse and recovery of marine fishes. Nature, 406(16798), 882.

Kelly, M., Xing, Y., and Lenhart, S. (2016). Optimal fish harvesting for a population modeled by a nonlinear parabolic partial differential equation. Natural Resource Modeling, 29, 36-69.

Langebrake, J., Lambert, L., Osenberg, C., and Leenheer, P. D. (2012). Differential movement and movement bias models for marine protected areas. Journal of Mathematical Biology, 64(4), 667-696.

Lester, S., Halpern, B., Grorud-Colvert, K., Lubchenco, J., Ruttenberg, B., Gaines, S., Airame, S., and Warner, R. (2009). Biological effects within notake marine reserves: A global synthesis. Marine Ecology Progress Series, 384, 33-46. 
Lockwood, D., Hastings, A., and Botsford, L. (2002). The effects of dispersal patterns on marine reserves: Does the tail wag the dog? Theoretical Population Biology, 61, 297-309.

Maciel, G. and Lutscher, F. (2013). How individual movement response to hapitat edge effects population persistence and spatial spread. The American Naturalist, 182, 42-52.

Maciel, G. and Lutscher, F. (2015). Allee effects and population spread in patchy landscapes. Journal of Biological Dynamics, 9(1), 109-123.

Maciel, G., Cosner, C., Cantrell, R., and Lutscher, F. (submitted). Evolutionarily stable movement strategies in reaction-diffusion models with edge behavior. Journal of Mathematical Biology.

Millar, R. (1992). Estimating the size-selectivity of fishing gear by conditioning on the total catch. Journal of the American Statistical Association, 87(420), 962-968.

Moberg, E., Shyu, E., Herrera, G., Lenhart, S., Lou, Y., and Neubert, M. (2015). On the bioeconomics of marine reserves when dispersal evolves. Natural Resource Modeling, 28, 456-474.

Moeller, H. and Neubert, M. (2013). Habitat damage, marine reserves, and the value of spatial management. Ecological Applications, 23, 959-971.

Mosquera, I., Côté, I., Jennings, S., and Reynolds, J. (2000). Conservation benefits of marine reserves for fish populations. Animal Conservation, 4, 321-332.

Neubert, M. (2003). Marine reserves and optimal harvesting. Ecology Letters, 6(9), 843-849.

Neubert, M. and Caswell, H. (2000). Density-dependent vital rates and their population dynamic consequences. Journal of Mathematical Biology, 41, $103-121$.

Olsgard, F., Schaanning, M., Widdicombe, S., Kendall, M., and Austen, M. (2008). Effects of bottom trawling on ecosystem functioning. Journal of Experimental Marine Biology and Ecology, 366(1-2), 123-133. 
Ovaskainen, O. and Cornell, S. (2003). Biased movement at a boundary and conditional occupancy times for diffusion processes. Journal of Applied Probability, 40, 557-580.

Reeve, J. and Cronin, J. (2010). Edge behaviour in a minute parasitic wasp. Journal of Animal Ecology, 79, 483-490.

Roberts, C., Bohnsack, J., Gell, F., Hawking, J., and Goodridge, R. (2001). Effects of marine reserves on adjacent fisheries. Science, 294(5548), 19201923.

Schultz, C. and Crone, E. (2001). Edge-mediated dispersal behavior in a prairie butterfly. Ecology, 82, 1879-1892.

Tsikliras, A. and Polymeros, K. (2014). Fish market prices drive overfishing of the big ones. PeerJ, 2, e638.

White, J. (2015). Marine reserve design theory for species with ontogenetic migration. The Royal Society, 11(1), 20140511-20140511.

Yurk, B. and Cobbold, C. (2018). Homogenization techniques for population dynamics in strongly heterogeneous landscapes. Journal of Biological Dynamics., 12, 171-193. 